\title{
Positional Order in Langmuir Monolayers
}

\author{
V. M. Kaganer,* G. Brezesinski, and H. Möhwald \\ Max-Planck-Institute of Colloids and Interfaces, D-12489 Berlin, Germany \\ P. B. Howes and K. Kjaer \\ Physics Department, Risø National Laboratory, DK-4000 Roskilde, Denmark
}

(Received 9 June 1998)

\begin{abstract}
We find that a structural solid-solid phase transition in a two-dimensional Langmuir film is accompanied by strong positional disorder. Specifically, we find by a grazing-incidence x-ray diffraction experiment that in monolayers of octadecanol both the hexagonal phase $L S$ and the centered rectangular (distorted hexagonal) phase $S$ have algebraic decay of positional correlations. The positional disorder is evidenced by variations of the exponent $\eta$, which drastically increases at the transition. The disorder is attributed to elastic distortions around pretransitional fluctuations. [S0031-9007(98)08060-0]

PACS numbers: 68.18.+p, 64.70.Md, 68.10.-m, 68.35.Rh
\end{abstract}

Positional order in two-dimensional systems is mostly studied on the simplest example of a monatomic layer whose atoms do not possess internal degrees of freedom. Algebraic decay of positional correlations at any nonzero temperature is the well-known consequence of the dimensionality of the system [1]. Langmuir monolayers (insoluble monolayers on a liquid surface) exhibit ordering of the short axes (backbone planes) of the molecule chains. This involves the coupling between the orientational degrees of freedom of individual molecules and their positional order [2]. The present x-ray diffraction study of the $L S-S$ transition in monolayers of octadecanol shows that the structural phase transition from the 2D hexagonal phase $L S$ to the centered rectangular (distorted hexagonal) phase $S$ is accompanied by strong positional disorder. Variations of the exponent $\eta$ of the algebraic decay are noticed as far as $20^{\circ} \mathrm{C}$ above the transition. On lowering the temperature, a continuous increase of $\eta$ to very large values $(\eta>2)$ results. The disorder is attributed to elastic distortions around pretransitional $S$-type fluctuations in the $L S$ phase.

We determine the correlation function of positional order by directly processing the measured peak profiles, without a fit to a presupposed model. This is possible because the monolayer is a two-dimensional powder of domains. The average of the structure factor over different domain orientations singles out the correlation function in the direction of the diffraction vector. We find algebraic decay of positional correlations, so that we must treat both phases, $L S$ and $S$, as $2 \mathrm{D}$ solids on the length scale available in present experiment $(<800 \AA)$, in contrast to their usual treatment-due to the width of the diffraction peaks which exceed the resolution limit and to the small viscosity of the $L S$ phase-as fluids. Hence, the transition $L S-S$ is treated as a $2 \mathrm{D}$ solid-solid structural phase transition. The effects of dislocationmediated melting are considered irrelevant to the problem because of a too low temperature or large dislocation core energies.
The study was performed on octadecanol [3] whose phase diagram is well established [4]. The diffraction measurements were performed in the untilted phases at a surface pressure of $18 \mathrm{mN} / \mathrm{m}$, well above the tilting phase transition. The tilted state of the octadecanol monolayers has been studied previously [5]. The grazing-incidence $\mathrm{x}$-ray diffraction measurements were performed at the liquid surface diffractometer on the undulator beam line BW1 at HASYLAB, DESY, Hamburg. The horizontal resolution, given mainly by a Soller collimator placed in front of a vertical position-sensitive detector, was measured by scanning the beam specularly reflected from the water surface. The shape of the resolution function was found to be very close to a Gaussian with a full width at half maximum (FWHM) of $0.0082 \AA^{-1}$, giving rise to a real-space resolution $2 \pi / \mathrm{FWHM} \approx 770 \AA$.

Figure 1 (left) shows the measured diffracted intensities corrected for the Lorentz factor, effective area and polarization, integrated over the vertical component, $Q_{z}$, of the scattering vector, and with a linear background subtracted. The present data are in agreement with the $\mathrm{x}$-ray diffraction study of the $L S-S$ transition in heneicosanoic acid monolayers [6] as well as with our previous study of octadecanol [5(a)] at temperatures below and well above the transition. However, the observation [5(a),6] of two overlapping broad peaks ( $L S_{I}$ phase) at intermediate temperatures was not reproduced. In Ref. [5(a)], the measurements were performed during continuous heating of the monolayer. These observations probably indicate a nonequilibrium nature of this diffraction pattern. Strong kinetic effects, with relaxation from a rectangular to a hexagonal structure within tens of minutes, have been reported for this part of the phase diagram [7]. The temperature variation of the diffraction pattern, Fig. 1, is in agreement with a first order phase transition $L S-S$ at $8{ }^{\circ} \mathrm{C}$, cf. Ref. [4]. Below this temperature (in phase $S$ ), one observes two diffraction peaks characteristic of a centered rectangular unit cell. Above $8{ }^{\circ} \mathrm{C}$ (phase $L S$ ) there is only one peak due to a hexagonal arrangement of the 
molecules. This peak becomes sharper with increasing temperatures, as was already observed in previous studies of Langmuir monolayers [5(a),6] as well as of a closely related bulk system, the 3D crystals of $n$-alkanes [8].

The averaging of the scattered intensity due to a two-dimensional powder of randomly oriented domains considerably simplifies the analysis of the positional correlations. Consider a purely two-dimensional problem with the scattering vector, $\mathbf{Q}$, in the monolayer plane. The structure factor, $s(\mathbf{Q})$, for the scattering by an individual domain in the vicinity of a reciprocal-lattice vector, $\mathbf{Q}_{0}$, can be represented as a Fourier transform, $s(\mathbf{Q})=\int G(\mathbf{r}) \exp (i \mathbf{q} \cdot \mathbf{r}) d^{2} r$, of the correlation function, $\quad G(\mathbf{r}) \equiv G_{\mathbf{Q}_{0}}(\mathbf{r})=\left\langle\exp \left\{i \mathbf{Q}_{0} \cdot[\mathbf{u}(\mathbf{r})-\mathbf{u}(0)]\right\}\right\rangle$, where $\mathbf{u}(\mathbf{r})$ are displacements of the atoms from their positions in the ideal lattice and $\mathbf{q}=\mathbf{Q}-\mathbf{Q}_{0}$. The average over domain orientations is equivalent to integration of $s(\mathbf{Q})$ over a circle of radius $Q$. The scattered intensity is concentrated in the vicinity of the reciprocal-lattice point and the integration over the circle can be replaced by that along the line perpendicular to $\mathbf{Q}_{0}$. Hence, one obtains the powder-averaged structure factor $S(q)=\int s(\mathbf{Q}) d q_{\perp}$ as a one-dimensional integral,

$$
S(q)=\int G(x) \exp (i q x) d x,
$$

where $q=Q-Q_{0}$ is the wave vector measured in the powder diffraction pattern, $x$ is the coordinate along $\mathbf{Q}_{0}$, and $G(x)$ is the correlation function $G(\mathbf{r})$, with the coordinate perpendicular to $\mathbf{Q}_{0}$ equal to zero. The measured intensity, $I(q)$, is the convolution of the structure factor, $S(q)$, with the resolution function, $R(q), I(q)=$ $S(q) \otimes R(q)$. Then, Fourier transforming this equation, one obtains the correlation function as $G(x)=\tilde{I}(x) / \tilde{R}(x)$, where the tilde denotes Fourier transforms of the corresponding functions. Thus, the correlation function in the direction of the diffraction vector (with resolution effects deconvolved) can be directly determined from the powder diffraction experiment, as shown in Fig. 1 (right).

In the range from tens to hundreds of $\AA$, the correlation functions in Fig. 1, plotted on a double-logarithmic scale, are close to straight lines and hence support a powerlaw decay, rather than an exponential decay which would be expected for hexatic phases (replotted on a singlelogarithmic scale, these lines are far from straight). The only exception is the correlation function at $8.6{ }^{\circ} \mathrm{C}$, close to the transition, which can be equally well fitted to a power decay or to an exponential decay. There is no evidence for a characteristic length (e.g., a "crystallite size") which would restrict the algebraic decay from the large $x$ side. The correlation functions are characterized by the lengths, $L$, of the order of $10 \AA$, which restrict the power law at small $x$. Hence the correlation functions may be conveniently interpolated by the function,

$$
G(x)=\left[1+(x / L)^{2}\right]^{-\eta / 2},
$$
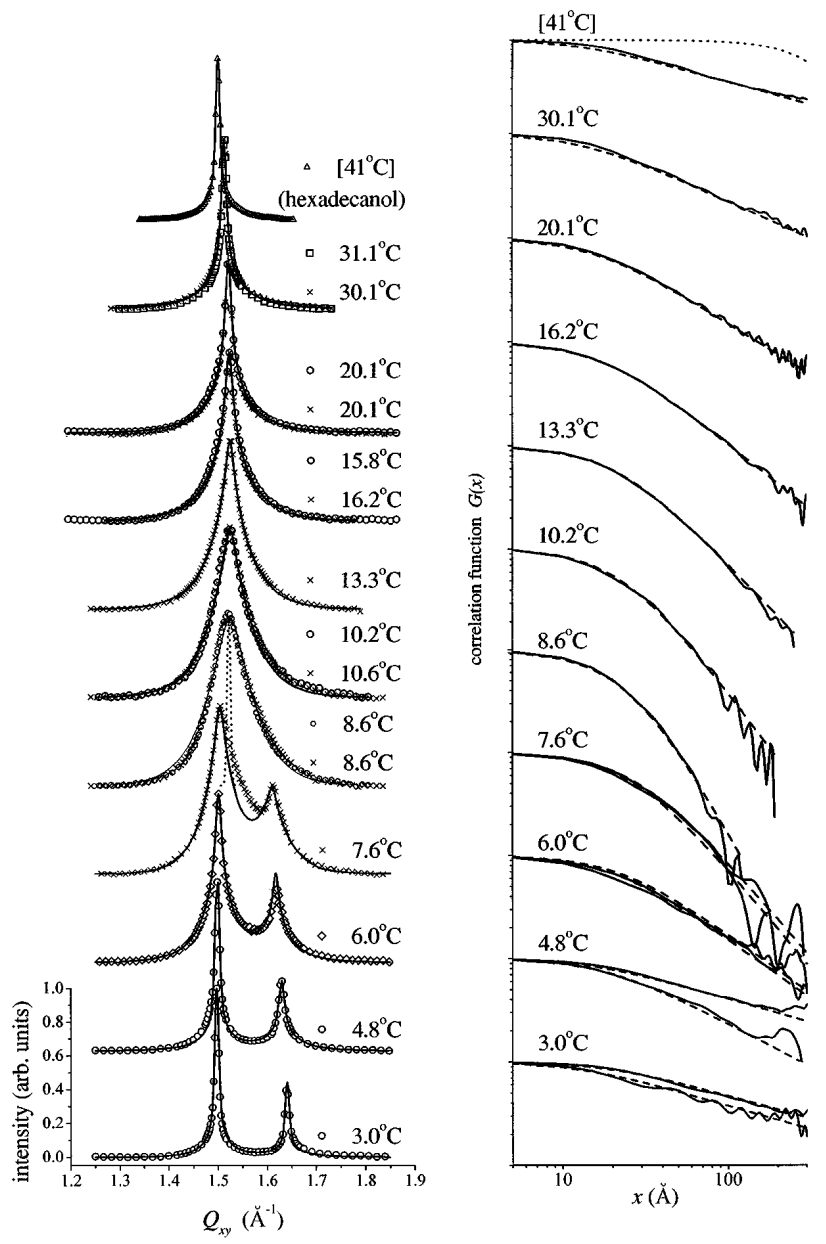

FIG. 1. X-ray diffraction peaks (left) and correlation functions (right) for octadecanol monolayers at the surface pressure $18 \mathrm{mN} / \mathrm{m}$. Different symbols correspond to the data from monolayers prepared in different ways. The circles denote the monolayer deposited at $3{ }^{\circ} \mathrm{C}$, compressed to $18 \mathrm{mN} / \mathrm{m}$, expanded to zero surface pressure, and compressed again to $18 \mathrm{mN} / \mathrm{m}$ at a new temperature after heating. The compression-expansion cycles were repeated on the same monolayer 11 times on heating from 3.0 to $20.1^{\circ} \mathrm{C}$. The crosses denote the data due to another monolayer which was compressed to $18 \mathrm{mN} / \mathrm{m}$ at $7.6^{\circ} \mathrm{C}$ and then heated to $30.1^{\circ} \mathrm{C}$ without expansion. Squares denote a monolayer deposited and first compressed at $31.1^{\circ} \mathrm{C}$. Hence, the diffraction peaks are essentially independent of the way in which the monolayer was prepared. The triangles denote the monolayer of hexadecanol at $31.7^{\circ} \mathrm{C}$, which corresponds approximately to the state of the octadecanol monolayer at $42^{\circ} \mathrm{C}$, due to the shortening of the chain by two methylene groups. The full lines over the peaks are the fits to the structure factor (3) convolved with the Gaussian approximation to the resolution function. The dotted line shows the resolution function. The widths of all peaks exceed the resolution limit. On the right, full lines present Fourier transforms of the measured peaks, divided by the Fourier transform of the resolution function (shown by the dotted line). When two diffraction peaks are observed due to a centered rectangular unit cell, they are processed separately and the correlation functions in directions of the corresponding diffraction vectors are determined. Dashed lines are fits to Eq. (2) with the same values of $\eta$ and $L$ as the fits of the peaks on the left. 
which contains a characteristic size $L$ and exhibits algebraic decay $G(x) \propto 1 / x^{\eta}$ for $x \gg L$. The functional form of Eq. (2) is just a convenient interpolation and there is no physical meaning behind it; it helps only to characterize the correlation functions by the appropriate parameters, $L$ and $\eta$. An advantage of Eq. (2) is the possibility of performing the Fourier transformation analytically,

$$
S(q)=|q|^{(\eta-1) / 2} K_{(\eta-1) / 2}(L|q|),
$$

where $K_{m}(z)$ is a modified Bessel function. Equation (3) provides a convenient interpolation formula describing (after convolution with the resolution function) the shape of the diffraction peak. The curves in the left column of Fig. 1 were obtained by numerically convolving Eq. (3) with the Gaussian approximation for the resolution function. The structure factor (3) may be used to fit the peaks when the scatter of the experimental data prevents the direct application of the Fourier transformation to obtain the correlation function. We tried both to fit the peak profiles to Eq. (3) (Fig. 1, left) and the correlation functions obtained by Fourier transformation of the peaks to Eq. (2) (Fig. 1, right) and found coincidence of the results.

Figure 2 presents temperature dependencies of the exponent, $\eta$, and the length, $L$. When two peaks are observed and processed separately (in the phase $S$ ), two values result, and their mean is shown in Fig. 2. Both parameters have maxima at the temperature of the first order transition $L S-S\left(8^{\circ} \mathrm{C}\right)$. The length, $L$, is comparable with the thickness of the monolayer $(\approx 22.5 \AA)$ and the simplest interpretation of this parameter could be as the crossover from two-dimensional to three-dimensional behavior. The systematic temperature variation of $L$ indicates another origin of this parameter, though. Note that $L$ varies from about three to five lattice spacings $(a \approx 4.8 \AA$ ).

For scattering due to lattice distortions, regardless of their nature, the exponent of algebraic decay is proportional to the square of the diffraction vector, $\eta \propto Q_{0}^{2}$. This dependence was verified by measuring the higherorder diffraction peaks in the hexagonal phase $L S$; Fig. 3. Good agreement of the calculated structure factors with
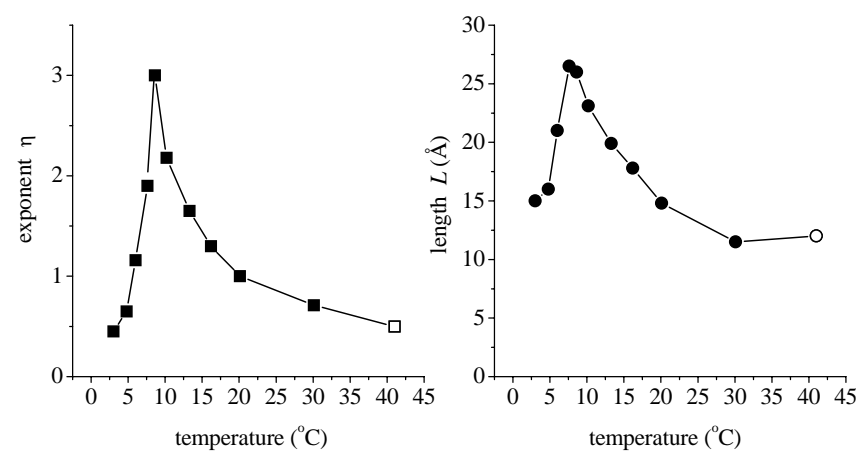

FIG. 2. Temperature dependencies of the exponent $\eta$ of the algebraic decay and the length $L$ determined from the fits to the peaks shown in Fig. 1. the data confirms that the broadening is caused by lattice distortions.

The long-wavelength thermal phonons in a twodimensional solid are the well-known source of the algebraic decay of positional correlations [1]. The theory of dislocation-mediated melting imposes a limit [9] $\eta<1 / 3$ on the exponent. If thermal phonons were considered as the only source of the algebraic decay, the exponents exceeding this limit might be due to a very high energy of nucleation of dislocation pairs making the dislocation-mediated melting scenario irrelevant [10]. Then the value of $\eta$ can be used to determine the shear modulus of the 2D solid, provided the compression modulus $K$ is measured independently. Our measurements at $30{ }^{\circ} \mathrm{C}$ give $K=3.45 \mathrm{~N} / \mathrm{m}$, in agreement with data on decanol [10] and behenic acid [11] monolayers, obtained in similar ways. Then one obtains a small ratio of the shear modulus to the compression modulus: $\mu / K \approx 0.03$, compared with the value $\mu / K \approx 0.04$ for the decanol monolayer at its melting transition obtained in the same way [10]. One can expect smaller ratios $\mu / K$ closer to the transition $L S-S$, because of the increase in $\eta$. Small values of the shear modulus may be related to the small viscosity of the phase $L S$ which gave the name ("superliquid") to this phase [12].

The relevance of the phase transition $L S-S$ to the temperature dependence of the parameters $\eta$ and $L$, which is evident from Fig. 2, suggests the order parameter fluctuations close to the transition as the most plausible source of the x-ray scattering. The fluctuations with the structure of the phase $S$ cause elastic distortions in the surrounding matrix, since the centered rectangular unit cell of the phase $S$ differs from the hexagonal unit cell of the phase $L S$ by a rather large $(\approx 9 \%)$ distortion. A closely related mechanism, scattering by sufficiently large domains of the rectangular phase, was discussed by Sirota (see Ref. [8]). We find that the domains remain small [containing $(L / a)^{2} \leq 30$ molecules even close to the
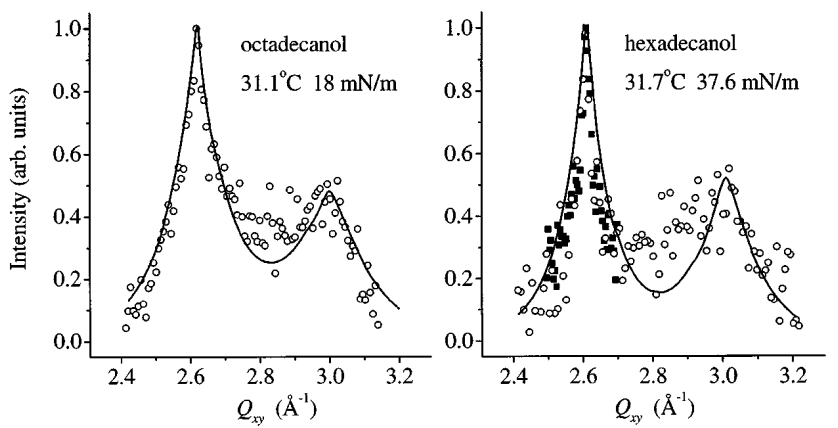

FIG. 3. The higher-order diffraction peaks of the hexagonal phase $L S$ in octadecanol and hexadecanol monolayers. Different symbols correspond to independent measurements. The lines are structure factors (3) with the peak positions calculated from the position of the corresponding low-order peak, the exponent $\eta$ scaled as $\eta \propto Q_{0}^{2}$, and the length $L$ given by the low-order peak. 
transition] and the peak broadening is due to distortions which they cause in the matrix. These distortions give rise to the algebraic decay whose exponent is not restricted by any universal value. Thus, the large value of the exponent can be realized, close to the structural phase transition, in the solid state of the two-dimensional system well below the dislocation unbinding temperature.

To calculate the correlation function of the translational order, we consider that the transition $L S-S$ is due to the herringbone ordering of the hydrocarbon chains and the lattice distortions are proportional to the square of the corresponding order parameter, $\varphi$ [2]. Then, one obtains in the harmonic approximation,

$$
G(x)=\exp \left\{-\eta \int \frac{d q}{q}\left[1-J_{0}(q x)\right]\left\langle\varphi^{2}\right\rangle^{2}\right\},
$$

where $J_{0}(z)$ is the Bessel function. Equation (4) differs from the well-known expression for the long-wavelength thermal phonons [13] only by the last multiplier which is due to distortions proportional to $\left\langle\varphi^{2}\right\rangle$. In the harmonic approximation one finds $[1]\left\langle\varphi^{2}\right\rangle=\left[1+(\xi q)^{2}\right]^{-1}$ (the amplitude of the fluctuations is incorporated in the constant $\eta$ ). The correlation length of fluctuations, $\xi$, provides the small-distance limit to the algebraic decay, $G(x) \propto x^{-\eta}$, for $x \gg \xi$. Calculating the integral (4) numerically and comparing it with Eq. (2), we find that, if one relates the characteristic length $L$ introduced by Eq. (2) to the correlation length of fluctuations $\xi$ as $\xi=L / 1.95$, the difference does not exceed a few $\%$. The analytical formula (2) is more convenient for purposes of peak fitting and we used it instead of Eq. (4). Landau theory predicts that $\xi$ and $\eta$ diverge at a second-order transition point as $\xi \propto t^{-1 / 2}$ and $\eta \propto t^{-2}$, where $t=T-T_{0}$. At a first-order transition, as in the present study, $T_{0}$ is not equal to the transition temperature and these parameters increase close to the transition but remain finite.

The positional correlations found in the present study correspond to quasi-long-range order in both phases $L S$ and $S$, in contrast to a common treatment of these phases as hexatics, based on the FWHMs of the peaks which exceed the resolution limit. The length limit available in the present experiment $(<800 \AA)$ does not allow one to judge the order (crystalline or hexatic) on longer distanced. The accessible length scale can be significantly increased (by a factor of $\geq 50$, cf. [10]) with the use of the new generation of synchrotron sources. We attribute the peak broadening with very large values of the exponent $\eta$ to elastic distortions around short-range fluctuations of the ordered phase above the transition (and corresponding disorder below the transition). Sirota [8] pointed out that, since we are dealing in regimes where a hexatic and a solid cannot necessarily be experimentally distinguished, the theory of phase transitions should not be too sensitive to such a distinction. The Landau theory of phase transitions in Langmuir monolayers, which was initially formulated in terms of the phase transitions between 2D-solid phases [14] and then reformulated in terms of the hexatic phases [2] using essentially the same free energy expansions, complies with this demand. However, the treatment of the phase $S$ as a 2D solid raises a question of the nature of the $S$-CS transition which was treated [2] as a crystallization of the mesophase. Changes in positional order at this transition can be studied, applying the data processing developed in the present paper, with molecular chains longer than that of octadecanol.

We are grateful to HASYLAB for beamtime. V. M. K. thanks Efim Kats for helpful discussions. This work was supported by Alexander von Humboldt Stiftung (V.M.K.), Deutsche Forschungsgemeinschaft (G.B.), Danish Dansync, and the E.U.'s TMR program (P.B.H., K. K.).

*Permanent address: Institute of Crystallography, Russian Academy of Sciences, 117333 Moscow, Russia.

[1] L.D. Landau and E. M. Lifshitz, Statistical Physics (Pergamon Press, Oxford, U.K., 1980), Pt. 1.

[2] V.M. Kaganer and E. B. Loginov, Phys. Rev. Lett. 71, 2599 (1993); Phys. Rev. E 51, 2237 (1995).

[3] $\mathrm{CH}_{3}\left(\mathrm{CH}_{2}\right)_{17} \mathrm{OH}$ (Fluka, Germany, purity $\left.>99.5 \%\right)$.

[4] G. A. Lawrie and G. T. Barnes, J. Colloid Interface Sci. 162, 36 (1994); C. Lautz and Th. M. Fischer, J. Phys. Chem. B 101, 8790 (1997).

[5] (a) G. Brezesinski, V. M. Kaganer, H. Möhwald, and P. B. Howes, J. Chem. Phys. 109, 2006 (1998); (b) C. Lautz, T. M. Fischer, M. Weygand, M. Lösche, P.B. Howes, and K. Kjaer, J. Chem. Phys. 108, 4640 (1998).

[6] M.C. Shih, T. M. Bohanon, J. M. Mikrut, P. Zschack, and P. Dutta, Phys. Rev. A 45, 5734 (1992).

[7] B. Lin, J. B. Peng, J. B. Ketterson, P. Dutta, B. N. Thomas, J. Buontempt, and S. A. Rice, J. Chem. Phys. 90, 2393 (1989).

[8] E. B. Sirota, Langmuir 13, 3849 (1997).

[9] D. R. Nelson and B.I. Halperin, Phys. Rev. B 19, 2457 (1979).

[10] C. Zakri, A. Renault, J.-P. Rieu, M. Vallde, B. Berge, J.-F. Legrand, G. Vignault, and G. Grübel, Phys. Rev. B 55, 14163 (1997).

[11] C. Fradin, J. Daillant, A. Braslau, D. Luzet, M. Alba, and M. Goldmann, Eur. Phys. J. B 1, 57 (1998).

[12] L. E. Copeland, W. D. Harkins, and G. E. Boyd, J. Chem. Phys. 10, 357 (1942).

[13] Y. Imry and L. Gunther, Phys. Rev. B 3, 3939 (1971).

[14] V. M. Kaganer and V. L. Indenbom, J. Phys. II (France) 3, 813 (1993). 